\title{
Describing Life to Define Death: A Cuban Perspective
}

\section{By Calixto Machado MD PhD DrSc}

Death-its essence, moment and certainty-has riveted the attention of religion, philosophy and science throughout the ages. To this day, debates among proponents of one definition or another are reflected in popular culture, belief systems and language itself.

In the mid-20 $0^{\text {th }}$ Century, however, a revolution occurred in the definition of death, provoked by the ethical and medical dilemmas inherent in the innovation of intensive care life-support mechanisms and the advent of organ transplantation involving cadaveric donors. Since then, global discussion has focused on the neurological: what part of the brain must be declared irreversibly dysfunctional for an individual's death to be declared?

But more is required. Science is challenged to encounter a single definition of death that integrates philosophical and ethical premises with a neurological understanding of brain function. So it must first answer the question: "What is the essence of human life"?, and then the question: "What conditions can be scientifically determined to be so inconsistent with human life as to render death?".

As a researcher and head of the Cuban commission established in 1992 to develop an applicable definition for determination and certification of death, I have joined those grappling with these questions for several decades. Over time, many of us have identified consciousness as the source of attributes essential to human existence, governing the organism's functions and making every person unique, with a singular life experience, interests, memories, and personality. Consciousness represents the integrated sum of cognitive, emotional, and higher psychological functions denoting understanding of one's existence and recognition of both internal and external worlds: the essence of human life.

Where there is even a vestige of consciousness, there is life. Thus, death should be defined conceptually as the irreversible loss of consciousness, the latter understood as that which generates essential human attributes and the highest level of control in the hierarchy of the body's integrated functions.[1]

Plum and Posner propose that consciousness includes two fundamental components: arousal (towards a wakeful state) and awareness (implying active contemplation and relation to self and surroundings).[2] The issue then is to identify where consciousness comes from, where these components are scientifically "located". We now know, of course, that love does not come "from the heart", but in fact from the brain. Yet the question remains: what part, or parts, of the brain determine love, determine consciousness itself? Research has led me to conclude that generation of consciousness does not pair handily with one or another superior or inferior brain structure-as some authors still arguebut instead presents an often cross-structural and inter-connected relationship. Consequently, for death to happen, irreversible loss of function must occur in the substantial interconnections among the brain stem, other subcortical structures and the cerebral cortex, which generate and integrate both components of consciousness.

This definition, upon recommendation of the above-mentioned commission, is the basis for Public Health Resolution 90 published in 2001, providing a legal framework for regulating determination and certification of death in Cuba.[3] That is, while the Resolution includes as "true signs of death" the irreversible absence of respiratory and circulatory functions, as well as presence of port-mortem changes, the commission concluded that an individual's death can only be defined in terms of an "irreversible loss of brain function". Put another way, there is only one death. This can be better understood observing that cardiorespiratory failure only provokes death when it is sufficiently prolonged for ischemia and anoxia to destroy brain function.

Where there is even a
vestige of consciousness,
there is life....death (is)
the irreversible loss of
consciousness

(applying classical cardiocirculatory and respiratory criteria); forensic circumstances (applying cadaveric signs of death); and with intensive-care external life support (applying criteria determining brain death, including a battery of multimodal evoked potentials and electroretinography to facilitate early diagnosis of brain death).

Additional aspects of the Resolution are worth noting, since they reflect particular scientific, legal and ethical points of view defended by the commission, but not necessarily shared internationally. First, unlike most laws and regulations in other countries, it purposefully does not link brain death to organ transplantation, emphasizing an ethical and clinical understanding that the two should be treated separately.

Second, highly relevant to the determination of death is the fact that the Cuban legal and clinical guidelines are expressed in a ministerial resolution rather than an act of parliament. Once again, this decision was deliberate, mindful that the "irreversibility" of brain function loss-critical for determining death-is a term whose meaning fluctuates with advances in science and technology. Unlike a law's straitjacket, a resolution encourages continual re-examination in light of new discoveries.

And that brings us to the mission of medicine itself: it is hoped, of course, that what is irreversible today might become reversible tomorrow, that consciousness lost can be recovered. In that context, we can also hope that our challenge in the future will be to ever more narrowly define death, based on expanded opportunities for life. 1 -

1. Machado C. Brain Death: A reappraisal. New York: Springer;2007. p. 121-6.

2. Plum F, Posner JB. The Diagnosis of Stupor and Coma. Philadelphia: FA Davis;1980.

3. Gaceta Oficial de la República de Cuba. Resolución No. 90 de Salud Pública. Edición Ordinaria del 21 de Septiembre de 2001. Havana: Ministry of Justice; 2001. 